\title{
Psoriasis in childhood
}

Psoriasis is a common probably polygenically determined papulosquamous skin disorder of unknown cause. An association has been found with the HLA antigen HLACW6. The risk for first degree relatives of an isolated case is at least $10 \%$ but children of two psoriatic parents have around a $50 \%$ risk of being affected. ${ }^{1}$ It affects approximately $2 \%$ of the population of Europe and North America and is more common in white people. In childhood it is more common in girls. In a 1988 survey I found $31 \%$ of adult psoriatic patient remembered an onset before the age of 16 but in Scandinavia the figure is $45 \%$. $^{2}$ Ten per cent of a large American sample had an onset before the age of 10 years. ${ }^{3}$ Farber et al re-examined nine children with psoriasis in infancy 6-13 years later and found that seven still had recurrent psoriasis, although this was generally not severe. ${ }^{4}$ One of these children had psoriasis from birth and congenital psoriasis has been reported previously. ${ }^{5}$

In children guttate psoriasis occurs more frequently than in adults and appears abruptly, often a week or so after a streptococcal tonsillitis or other infection and may be widespread. The small papular lesions have characteristic overlying silvery scales. The guttate eruption usually persists for three to four months resolving spontaneously but it can persist for over a year. After an attack of guttate psoriasis it is usual for psoriasis of some type to recur within the next three to five years.

The Köbner phenomenon in which lesions appear along the site of injury may be seen in active psoriasis. Parents should be warned that skin trauma-for example, scratches or sunburn-may result in psoriasis at such trauma sites.

Psoriasis vulgaris, the plaque type of psoriasis, is also common in children; as well as plaques, ringed and geographic patches of varying sizes may occur. Plaques may be encircled by a clear pale zone, the ring of Woronoff. Scalp psoriasis is typified by patchy thick scaling areas and when scales are removed some hair may be lost but almost always regrows. Pityriasis amiantacea describes a condition in which scalp patches occur showing white asbestos-like scales, which cling firmly to the hair shafts as they emerge from the scalp and extend some distance along them; in children it is often psoriatic.

Nail changes are seen particularly in chronic psoriasis and are uncommon in children, but onycholysis of finger nails (separation of the distal nail plate from the nail bed) and pitting may be seen and occasionally nail changes are seen with minimal skin involvement.

Pustular psoriasis is rare in children but abuse of the more potent topical corticosteroids in the treatment of ordinary psoriasis and sometimes their withdrawal may precipitate this form. ${ }^{6}$ However, in children psoriasis may present as a pustular acrodermatitis involving one or two fingers and this may coexist with or be followed later by typical psoriasis elsewhere.

Psoriatic arthropathy is rare in children but may precede the onset of skin lesions. It may be initially misdiagnosed as juvenile rheumatoid arthritis but systemic manifestations associated with juvenile rheumatoid arthritis are usually absent. $^{7}$

As regards treatment most children can be managed as outpatients. Bland preparations such as aqueous cream or coal tar solution $2 \%$ in emulsifying ointment are recommended for guttate psoriasis. Coal tar preparations are useful for psoriasis vulgaris and scalp psoriasis. Sunlight and ultraviolet light $B$ have a beneficial effect in chronic psoriasis in most individuals but are contraindicated in acute guttate or other acute psoriasis.

A common time honoured regimen for plaque psoriasis is a tar bath followed by exposure to ultraviolet light B and then application of dithranol in Lassar's zinc paste with salicylic acid. Dithranol may irritate the skin and treatment should start with a low concentration. Patients/parents should be informed of the temporary brown skin staining dithranol produces. Short contact dithranol, preferably in the form of a cream (for example, Dithrocream, (Dermal)) applied for 20-60 minutes in the evening before a bath and bed is a modern effective treatment which allows freedom from clothes staining applications during the school day.

Sparing use of topical corticosteroids of mild or moderate potency does have a place in the treatment of face, ear, flexural, and genital lesions. Such preparations with their ability to suppress inflammation may sometimes also be used in combination with tar over limbs and trunk in irritant psoriasis.

An interesting recent treatment, so far limited to adult experience, is calcipotriol, a synthetic vitamin D analogue, applied in ointment form. Early evidence suggests this to be a safe effective preparation in moderate psoriasis, ${ }^{8}$ and one of its actions is known to be suppression of epidermal keratinocyte proliferation, which is appreciably increased in psoriasis.

Although diet has no proved relationship to psoriasis oral fish oils may at times produce modest benefit. ${ }^{9}$ Methotrexate $^{10}$ and oral synthetic retinoids ${ }^{11}$ would be indicated only in rare uncontrolled erythrodermic psoriasis or generalised pustular psoriasis. Photochemotherapy (phototoxic psoralens used in combination with ultraviolet light A), long term use of which causes an increased risk of skin cancer, would also not be indicated in the routine management of childhood psoriasis. It is doubtful whether any place will be found in childhood psoriasis for cyclosporin, a potent immunosuppressant that is considerably nephrotoxic, but longer term adult studies will determine its safety. ${ }^{12}$

It is important to discuss with the parents of a psoriatic child all important aspects of the disease. Avoidance of emotional stress, where possible, may prevent a flare of eruption. Emphasis should be placed on spontaneous improvement and likely long remissions with treatment. The non-contagious nature of psoriasis should also be mentioned. Some families may wish to discuss the disorder with others and may wish to join the Psoriasis Association ( 7 Milton Street, Northampton NN2 7JG).

Royal Liverpool Children's Hospital,

JULIAN VERBOV

Myrtle Street,

Liverpool $L 77 D G$ 1 Harper PS. The skin. Practical genetic counselling. 3rd Ed. London: Wright,
1988:195. 
2 Nyfors A, Lemholt $K$. Psoriasis in children. A short review and a survey of 245 cases. Br 7 Dermatol 1975;92:437-42.

3 Farber EM, Nall ML. The natural history of psoriasis in 5600 patients. Dermatologica 1974;148:1-18.

4 Farber EM, Mullen RH, Jacobs AH, Nall L. Infantile psoriasis: a follow-up study. Pediatr Dermatol 1986;3:237-43.

5 Lerner MR, Lerner AB. Congenital psoriasis: report of three cases. Arch Dermatol 1972;105:598-601.

6 Boxley JD, Dawber RPR, Summerly R. Generalised pustular psoriasis on withdrawal of clobetasol propionate ointment. BMF 1975;ii:255-6.

7 Perlman SG. Psoriatic arthritis in children. Pediatr Dermatol 1984;1:283-7.
8 Kragballe K, Gjertsen BT, de Hoop D, et al. Double-blind, right/left comparison of calcipotriol and betamethasone valerate in treatment of psoriasis vulgaris. Lancet 1991;337:193-6.

9 Burton JL. Dietary fatty acids and inflammatory skin disease. Lancet 1989;i:27-31.

10 Lowe NJ. Psoriasis. Semin Dermatol 1988;7:43-7.

11 Rosińska D, Wolska H, Jablonska S, Konca I. Etretinate in severe psoriasis of children. Pediatr Dermatol 1988;55:266-72.

12 Korstanje MJ, van de Staak WJBM. Long-term treatment of psoriasis with cyclosporin A-side effects, minimal effective dose and cyclosporin blood levels. Clin Exp Dermatol 1991;16:8-10. 